\title{
Application of Bee Evolutionary Genetic Algorithm to Maximum Likelihood Direction-of-Arrival Estimation
}

\author{
Xinnan Fan, ${ }^{1,2}$ Linbin Pang, ${ }^{1,3}$ Pengfei Shi $\mathbb{D}^{1},{ }^{1}$ Guangzhi Li, ${ }^{1}$ and Xuewu Zhang ${ }^{1}$ \\ ${ }^{1}$ College of IOT Engineering, Hohai University, Changzhou 213022, China \\ ${ }^{2}$ Jiangsu Provincial Collaborative Innovation Center of World Water Valley and Water Ecological Civilization, \\ Nanjing, Jiangsu 211100, China \\ ${ }^{3}$ College of Computer and Information, Hohai University, Nanjing 211100, China \\ Correspondence should be addressed to Pengfei Shi; flyshn@hotmail.com
}

Received 9 September 2018; Revised 25 January 2019; Accepted 12 February 2019; Published 25 February 2019

Academic Editor: George S. Dulikravich

Copyright (C) 2019 Xinnan Fan et al. This is an open access article distributed under the Creative Commons Attribution License, which permits unrestricted use, distribution, and reproduction in any medium, provided the original work is properly cited.

\begin{abstract}
The maximum likelihood (ML) method achieves an excellent performance for DOA estimation. However, its computational complexity is too high for a multidimensional nonlinear solution search. To address this issue, an improved bee evolutionary genetic algorithm (IBEGA) is applied to maximize the likelihood function for DOA estimation. First, an opposition-based reinforcement learning method is utilized to achieve a better initial population for the BEGA. Second, an improved arithmetic crossover operator is proposed to improve the global searching performance. The experimental results show that the proposed algorithm can reduce the computational complexity of ML DOA estimation significantly without sacrificing the estimation accuracy.
\end{abstract}

\section{Introduction}

Direction-of-arrival (DOA) estimation is not only an important process for many military and civilian applications but also a basic function of certain equipment such as sonar and torpedoes [1]. The performance of estimation precision and speed directly influence the performance of such equipment. This has been a hot research topic for decades, and researchers have proposed some useful methods. In general, these methods can be divided into three types [2]: conventional beamforming (CBF), subspace-based, and maximum likelihood (ML) methods [3].

The CBF algorithm is the earliest DOA estimation method based on an array and can be traced back to World War II $[2,4]$. The algorithm is a natural extension of classical Fourier-based spectral analysis on sensor array data. The angular resolution of the $\mathrm{CBF}$ algorithm is limited by the array physical aperture and cannot distinguish between two spatial targets within a beam width, which is called the Rayleigh resolution limit. Therefore, overcoming the limitation imposed by the array physical aperture is an important research direction and promotes the development of spatial spectrum estimation.
The earliest and well-known high-resolution DOA estimation algorithms are multiple-signal classification [5] and the estimation of signal parameters via the rotational invariance technique [6]. They are both representative of subspacebased algorithms. They can achieve simpler but suboptimal solutions, which decompose the observation space into signal and noise subspaces. They are widely known for their good angle estimation performance. However, conventional subspace techniques necessitate the eigenvalue decomposition or singular value decomposition of the covariance matrix to estimate the signal and noise subspaces; thus, a huge computational complexity is presented, particularly when the number of sensors is large such as in large towed sonar arrays.

ML estimation is simple and achieves a good estimation performance, especially in asymptotic estimation $[7,8]$. This method is often able to achieve the Cramér-Rao bound $(\mathrm{CRB})$, and this peculiarity is fairly ideal in many DOA estimation tasks, where a high estimation accuracy is required but the signal-to-noise ratios (SNRs) are usually low. The ML estimation algorithm performs well, and it can estimate not only incoherent sources but also coherent sources. However, the likelihood function of the ML estimation algorithm is a multidimensional nonlinear function, which results in a high 
computational cost. Thus, reducing the number of calculations has become an urgent problem in the development of ML estimation algorithms.

In past decades, to reduce the computational complexity, many alternative multidimensional searching methods have been proposed [9-11], such as the Newton-Gauss method, alternating projection method, space-alternating generalized expectation-maximization method, and method of direction estimation (MODE). Unfortunately, the application of these methods remains restricted due to their drawbacks. In the alternating projection method, the multidimensional search is converted into a unidimensional search; however, the convergence becomes slow when the source number increases. To obtain reliable and accurate estimates, detailed knowledge of the response of the measurement system is required in the space-alternating generalized expectation-maximization method. Meanwhile, the computational complexity is not reduced because of the iteration process. In addition, in most MODE-based methods, an eigendecomposition of the data covariance matrix is still required. Moreover, all MODEbased methods are restricted by a threshold, which is required in the method. The method performs well only when the SNR or snapshot number exceeds the threshold value; otherwise, the performance decreases greatly.

In recent years, intelligent algorithms based on bionics have seen rapid development and have been used widely. Various researchers have attempted to use these algorithms to solve the multidimensional nonlinear searching problem in ML DOA estimation. The genetic algorithm (GA) is one of the most powerful and popular global optimization tools. It is convenient for GA to choose a small population size and a low crossover fraction. However, this algorithm is limited by its precocity and slow convergence when population increased. This limitation may be surpassed by means of the implementation of parallel programming algorithms [12]. To improve the performance of GAs, several modified GAs, such as HeMI, ECG, and PGAs, have been proposed [1315]. However, the performances of the improved GAs are not satisfactory when some conditions are changed, such as more inputs, high feature dimensionality, or high computational complexity. With the development of bioinspired computing, increasingly more methods have been proposed and are being used for the DOA estimation optimization problems [16-20] such as the artificial bee colony (ABC) algorithm, particle swarm optimization (PSO) algorithm, differential evolution (DE) algorithm, and ant colony (ACO) algorithm.

The bee evolutionary genetic algorithm $[21,22]$ is an improvement on the GA. This technique introduces the excellent evolutionary characteristics of bees to emphasize the importance of the optimum individual in the population evolution and can converge to a global optimal solution quickly. In the BEGA, the optimum individual is selected as a queen bee from the population; then, a crossover operation is performed with each selected individual (drones). To avoid premature convergence, the BEGA introduces a random population, which is used to extend the searching area. The BEGA has been applied in many areas $[21,23,24]$ such as the packing problem and layout design. The BEGA is essentially

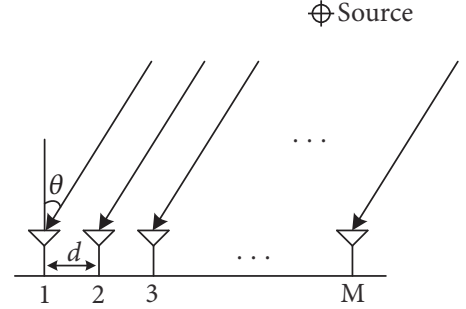

FIGURE 1: Sketch of an array receiving a signal.

a stochastic search optimization technique and performs well in solving nonlinear global optimal problems, making it possible to solve the computational complexity problem facing ML DOA estimation.

In this paper, we apply bee evolutionary genetic algorithm for ML DOA estimation. First, this paper converts the ML DOA estimation to a problem that the conventional BEGA can solve. This can reduce the computational complexity, but the estimation accuracy is not satisfactory. Therefore, this paper improves upon the conventional BEGA from two respects: the population initialization method and the crossover operator. The improved algorithm is called the IBEGA algorithm. The initial population provides an initial guess of the solutions. A good initial population can increase the convergence speed and lead to high-quality final solutions. An opposition-based reinforcement learning method is utilized to realize this goal. As mentioned above, the BEGA belongs to the GA family, and it is an improvement on the GA. For these types of algorithms, the crossover operator determines the global search performance. Due to the nonsatisfactory estimation accuracy of the conventional BEGA, this paper modifies the arithmetic crossover operator in the conventional BEGA. Finally, this paper conducts some simulations to verify the applicability of the IBEGA algorithm to ML DOA estimation. The simulations contain not only a comparison between the conventional BEGA and IBEGA algorithms but also a comparison among the IBEGA algorithm and other intelligent algorithms such as ABC, DE, and PSO. As a result, the computational complexity of ML DOA estimation can be greatly reduced without a loss of estimation accuracy.

The remainder of this paper is organized as follows. In Section 2, the signal model and ML DOA estimator are reviewed. In Section 3, the conventional BEGA algorithm and the proposed IBEGA algorithm are defined. The simulation results are shown in Section 4, and conclusions are drawn in Section 5 .

\section{Signal Model and ML DOA Algorithm}

2.1. Signal Model of Array. Assume that a uniform linear array is composed of $M$ elements and that its element gap is $d$. A sketch of the array receiving a signal from a far-field source is as shown in Figure 1.

Consider a uniform linear array receiving $P$ narrowband signals from a far field but ignoring element coupling, 
channel and band mismatch, etc. The array output data vector at time $k$ is

$$
\boldsymbol{X}(k)=\boldsymbol{A}(\theta) \boldsymbol{S}(k)+\boldsymbol{N}(k), \quad k=1,2, \ldots, L
$$

where $S(k) \in C^{P \times 1}$ is the complex amplitude vector of the source, $N(k) \in C^{M \times 1}$ is a complex vector of additive noise, $L$ is the snapshot number, and $\boldsymbol{A}(\theta)$ is an $M \times P$-dimensional array manifold matrix:

$$
\boldsymbol{A}(\theta)=\left[\begin{array}{llll}
\boldsymbol{a}_{1}\left(\theta_{1}\right) & \boldsymbol{a}_{2}\left(\theta_{2}\right) & \cdots & \boldsymbol{a}_{P}\left(\theta_{P}\right)
\end{array}\right]
$$

Here, $\boldsymbol{a}\left(\theta_{i}\right)$ is the steering vector:

$$
\boldsymbol{a}\left(\theta_{i}\right)=\frac{1}{\sqrt{M}}\left[\begin{array}{llll}
1 & e^{-j \varphi\left(\theta_{i}\right)} & \cdots & e^{-j(M-1) \varphi\left(\theta_{i}\right)}
\end{array}\right]^{T}
$$

where $\varphi\left(\theta_{i}\right)=(2 \pi d / \lambda) \sin \left(\theta_{i}\right) . \theta_{i} \in[-\pi / 2, \pi / 2]$.

The covariance matrix of the array snapshot data is

$$
\boldsymbol{R}=E\left\{\boldsymbol{X} \boldsymbol{X}^{H}\right\}=\boldsymbol{A} \boldsymbol{R}_{S} \boldsymbol{A}^{H}+\boldsymbol{R}_{N}
$$

where $\boldsymbol{R}_{S}$ is the signal covariance matrix and $\boldsymbol{R}_{N}$ is the noise covariance matrix.

In practice, only $L$ snapshot data sets are available. Thus, the covariance matrix $\boldsymbol{R}$ is instead the sample covariance matrix $\widehat{\boldsymbol{R}}$, which is

$$
\widehat{\boldsymbol{R}}=\frac{1}{L} \sum_{i=1}^{L} \boldsymbol{X} \boldsymbol{X}^{H}
$$

where $L$ is the number of snapshots.

2.2. Maximum Likelihood DOA Estimation. Suppose that the first order and second order of the snapshot data satisfy the following conditions:

$$
\begin{aligned}
& \boldsymbol{E}\left\{\boldsymbol{X}\left(k_{i}\right)\right\}=\boldsymbol{A}(\theta) \boldsymbol{S}\left(k_{i}\right) \\
& \boldsymbol{E}\left\{\left(\boldsymbol{X}\left(k_{i}\right)-\overline{\boldsymbol{X}}\left(k_{i}\right)\right)\left(\boldsymbol{X}\left(k_{j}\right)-\overline{\boldsymbol{X}}\left(k_{j}\right)\right)^{H}\right\}=\sigma^{2} \boldsymbol{I} \delta_{i j} \\
& \boldsymbol{E}\left\{\left(\boldsymbol{X}\left(k_{i}\right)-\overline{\boldsymbol{X}}\left(k_{i}\right)\right)\left(\boldsymbol{X}\left(k_{j}\right)-\overline{\boldsymbol{X}}\left(k_{j}\right)\right)^{T}\right\}=\mathbf{0}
\end{aligned}
$$

With (6), (7), and (8), we can know that the joint (conditional) probability density function of the $L$ snapshot data sets is

$$
f\left\{\boldsymbol{X}_{1}, \boldsymbol{X}_{2}, \ldots, \boldsymbol{X}_{L}\right\}=\prod_{i=1}^{L} \frac{1}{\operatorname{det}\left\{\pi \sigma^{2} \boldsymbol{I}\right\}} e^{-\left(1 / \sigma^{2}\right)\left\|\boldsymbol{X}_{i}-\boldsymbol{A}(\theta) S\right\|^{2}}
$$

where $\operatorname{det}\{\bullet\}$ is the determinant of the matrix and $\|\bullet\|$ is the Euclidean norm.

The negative logarithm of (9) is

$$
-\ln f=L \ln \pi+M L \ln \sigma^{2}+\frac{1}{\sigma^{2}} \sum_{i=1}^{L}\left\|\boldsymbol{X}_{i}-\boldsymbol{A}(\theta) \boldsymbol{S}\right\|^{2}
$$

where $f$ is a multidimensional nonlinear function of the unknown parameters $\theta, \sigma^{2}$, and $S$.
The deterministic ML DOA estimation of the unknown parameters $\sigma^{2}$ and $S$ from (10) is

$$
\begin{aligned}
\sigma^{2} & =\frac{1}{M} \operatorname{tr}\left\{\boldsymbol{P}_{A}^{\perp} \widehat{\boldsymbol{R}}\right\} \\
\widehat{\boldsymbol{S}} & =\boldsymbol{A}^{+} \boldsymbol{X}
\end{aligned}
$$

where $\operatorname{tr}\{\bullet\}$ is the trace of a matrix, $\boldsymbol{P}_{A}^{\perp}$ is the orthogonal projection matrix of the matrix $\boldsymbol{A}$, and $\boldsymbol{A}^{+}$is the pseudoinverse of the matrix $A$.

Substituting (11) and (12) back into (10), we obtain the ML estimation of the parameter $\theta$ :

$$
\widehat{\Theta}=\min _{\theta}\left\{\operatorname{tr}\left\{\boldsymbol{P}_{A}^{\perp} \widehat{\boldsymbol{R}}\right\}=\max _{\theta}\left\{\operatorname{tr}\left\{\boldsymbol{P}_{A} \widehat{\boldsymbol{R}}\right\}\right\}\right.
$$

where $\Theta=\left[\begin{array}{llll}\theta_{1} & \theta_{2} & \cdots & \theta_{P}\end{array}\right]^{T}$ and $\boldsymbol{P}_{\boldsymbol{A}}$ is the projection of the matrix $A$.

Grid search is one of the most accurate methods for finding the optima of likelihood functions. Its computational load depends on not only the grid size and searching range that we select but also the number of sources that we want to estimate. We can approximate the computational complexity $C_{G S}$ for calculating the likelihood function over a grid search as follows:

$$
C_{G S}=\left(\frac{r_{\max }-r_{\min }}{\text { Step }}\right)^{P} \times \Delta
$$

where $\left(r_{\max }, r_{\text {min }}\right)$ is the searching range, $\Delta$ is the number of single DOA calculations, Step is the distance between each grid point, and $P$ is the number of signal sources. Clearly, as the number of signal sources increases, the computational load will increase exponentially, which restricts the application of this approach.

\section{The Proposed Improved BEGA-Based ML DOA Estimation Algorithm}

Because the performance of the above-mentioned spectral searching ML DOA estimator is governed by the scanning grid size and the number of searching grids when implementing the high-resolution DOA estimation, the technique is time consuming, and the searching grid is not clear. Therefore, this paper applies the BEGA to maximize the likelihood function of the ML DOA estimator at each iteration.

In this section, first, the BEGA is introduced. Then, the ML DOA estimation is converted into a problem that the BEGA can solve. Finally, due to the unsatisfactory estimation accuracy of the conventional BEGA, this paper modifies it from two respects: the population initialization method and the crossover operator.

3.1. Bee Evolutionary Genetic Algorithm. The bee evolutionary genetic algorithm was proposed by Meng Wei et al. in 2006 [25]. The BEGA is similar to nature in that the queen bee, the fittest individual in a generation, crossbreeds with the other bees selected as parents from the original generation by a selection operator. Simultaneously, a random population is 


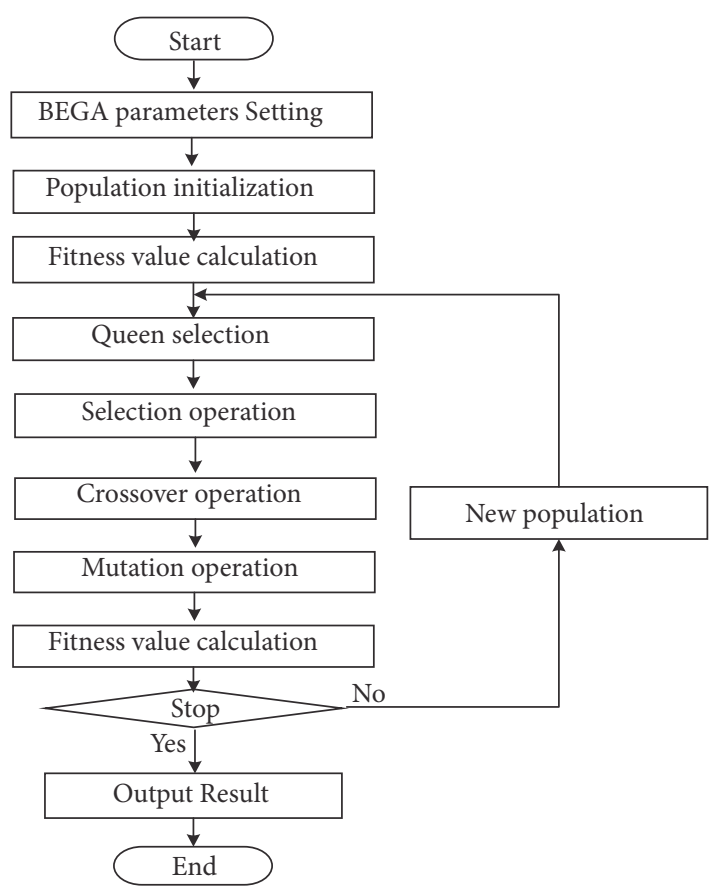

FIGURE 2: The main steps of the proposed BEGA algorithm.

also introduced as parents to extend the searching area. This enhances the exploration of the genetic algorithm. This is an excellent global optimization algorithm and has many advantages such as its low computational load, fast convergence, and small adjustment term. Therefore, it has been widely used to solve various types of optimization problems.

The basic ideas underlying the BEGA algorithm are presented below. First, the algorithm finds the optimal individual of the current population in each iteration. Then, it compares the optimal individual with the queen bee of the last generation, and the winner is selected as the current queen bee. Second, it selects $(l / 2) \gamma$ individuals from the current population and produces $(l / 2)(1-\gamma)$ new individuals randomly. $l$ is the population size, $\gamma$ is the selection ratio, with the latter's range being $[0,1]$. Third, it obtains the son generation population through the crossover and mutation operators from the queen bee and individuals that are produced in the second step. Fourth, the algorithm finds the optimal individual of the son generation population. If the fitness value of the optimal individual that is found from the son generation population is higher than the fitness value of the queen bee, the entire son generation population is used as the population of the next generation. Otherwise, the worst individual of the son generation population is replaced by the queen bee, and the modified son generation population is used as the population of the next generation.

Based on the above ideas, the main steps of the BEGA algorithm are presented in Figure 2.

3.2. Conventional BEGA for ML DOA Estimation. Based on the introduction in Section 3.1, the BEGA can be applied for ML DOA estimation as follows.
(1) Generate an initial population $G(t)=\left[\boldsymbol{\theta}_{1}, \boldsymbol{\theta}_{2}, \ldots, \boldsymbol{\theta}_{l}\right]$, $t=0$. $t$ denotes the current iteration number. Each $\boldsymbol{\theta}_{i}$ in $G(t)$ has $P$ dimensions, which means that $\boldsymbol{\theta}_{i}=\left[\theta_{i}^{1}, \theta_{i}^{2}, \ldots, \theta_{i}^{P}\right]$. The dimension number of each bee $\boldsymbol{\theta}_{i}$ is equal to the signal source number that must be estimated. In general, the initial population is generated by a uniform distribution function and can be expressed as follows:

$$
\begin{aligned}
\theta_{i}^{j}=r_{\text {min }}+\operatorname{rand}(0,1) \times & \left(r_{\text {max }}-r_{\text {min }}\right), \\
& i=1,2, \ldots, l ; j=1,2, \ldots, P
\end{aligned}
$$

where $\operatorname{rand}(0,1)$ is a random number generated in the interval $[0,1]$, which obeys a uniform distribution.

(2) Calculate the fitness values of the initial population and find the best individual as the initial queen Queen (0). We define a fitness function equal to the likelihood function of the ML DOA estimation as follows:

$$
\text { Fit }\left(\boldsymbol{\theta}_{i}\right)=\operatorname{tr}\left\{\left[A\left(\boldsymbol{\theta}_{i}\right)\left[A^{H}\left(\boldsymbol{\theta}_{i}\right) A\left(\boldsymbol{\theta}_{i}\right)\right]^{-1} A^{H} \boldsymbol{\theta}_{i}\right] \widehat{R}\right\}
$$

(3) Judge whether the stopping criterion is met. If the criterion is met, stop the iteration and output the result; otherwise, $t=t+1$, and continue. In this paper, the stop criterion uses the maximum iteration number criterion, which makes the judgment by checking whether the iteration number has reached the maximum number.

(4) Select $(l / 2) \gamma$ individuals from the population $G(t)$. This paper uses 'roulette wheel' selection as the selection operator; a detailed discussion of this selection type can be found in [26].

(5) Generate $(l / 2)(1-\gamma)$ individuals randomly. The generation method is the same as in Step (1).

(6) Queen $(t-1)$ performs the crossover operation with $l / 2$ of the individuals, which are selected from Step (3) and Step (4), to generate the population $C(t)=\left[\boldsymbol{\theta}_{i}^{A}, \boldsymbol{\theta}_{i}^{B}\right]$. The crossover operator uses the arithmetic crossover operator and can be expressed as follows [27]:

$$
\begin{aligned}
\boldsymbol{\theta}_{i}^{A} & =\alpha \times \text { Queen }+(1-\alpha) \times \boldsymbol{\theta}_{i} \\
\boldsymbol{\theta}_{i}^{B} & =(1-\alpha) \times \text { Queen }+\alpha \times \boldsymbol{\theta}_{i}
\end{aligned}
$$

where $\alpha$ is the crossover parameter, which is generally set to 0.5 .

(7) Apply the mutation operation to the population $C(t)$ to obtain the population $M(t)=\left[\boldsymbol{\theta}_{1}^{\prime}, \boldsymbol{\theta}_{2}^{\prime}, \ldots, \boldsymbol{\theta}_{l}^{\prime}\right]$. The mutation operator uses the 'Uniform mutation' method, which can be expressed as follows:

$$
\boldsymbol{\theta}_{\boldsymbol{i}}^{\prime}=\boldsymbol{\theta}_{\boldsymbol{i}}+\operatorname{rand}(0,1)
$$

(8) Calculate the fitness value of the population $M(t)$ and select an individual as Queen $_{\text {New }}$, whose fitness value is the maximum.

(9) If the fitness value of Queen ${ }_{N e w}$ is higher than that of Queen $(t-1)$, let Queen $(t)=$ Queen $_{\text {New }}$, and the $t$ th population $G(t)=M(t)$; otherwise, let Queen $(t)=$ Queen $(t-$ 1). Simultaneously, the worst individual in $M(t)$ is replaced by Queen $(t-1)$, and the modified population $M(t)$ is the $t$ th population $G(t)$.

(10) Go to Step (3) and start the next iteration. 
3.3. Improvements on Conventional BEGA for $M L D O A$ Estimation. Due to the unsatisfactory estimation accuracy, the conventional BEGA is improved from two aspects: the population initialization method and the crossover operator.

3.3.1. Improved Population Initialization Method. Population initialization is the starting point for population evolution and provides an initial guess of the solutions. Then, these initially guessed solutions are iteratively improved in the course of the optimization process until a stopping criterion is met. Generally, population initialization can affect the convergence speed and quality of the final solution [28]. A good population facilitates the convergence to good solutions, whereas a poor initial population can hinder the convergence.

As described above, (15) represents the common method for population initialization. The method employs pseudorandom number generators (PRNGs) to produce the initial population. The rationale behind this is that PRNGs can generate uniformly distributed samples [29], and thus, a population initialized using PRNGs tends to cover promising regions (containing global optima or good local optima) of the search space. Because the population size is always limited, the chance for a population to cover promising regions of the search space decreases with increasing range of the search space. Therefore, increasing the chance for a population to cover promising regions of the search space is important.

To obtain a better initial population, this paper utilizes an opposition-based reinforcement learning (OBRL) method [30] to modify the common population initialization method. The OBRL method is a multistep technique that uses a fitness function as a guideline for enhancing the initial population. First, the OBRL method generates a set of points called the original population. The original population can be generated using any initializer technique. Second, some simple heuristic rules are employed to produce another population with the same size in the last step. Finally, a subset of the union of both populations is selected based on their fitness values. Equation (19) shows the heuristic rule that produces an opposite population based on the original population:

$$
\boldsymbol{\theta}_{\boldsymbol{i}}^{\boldsymbol{O}}=\boldsymbol{r}_{\min }+\boldsymbol{r}_{\max }-\boldsymbol{\theta}_{\boldsymbol{i}}, \quad i=1,2, \ldots, l
$$

where $\boldsymbol{\theta}_{\boldsymbol{i}}^{\boldsymbol{O}}$ is the opposite individual of $\boldsymbol{\theta}_{\boldsymbol{i}}$ in the solution range $\left(r_{\min }, r_{\max }\right)$.

3.3.2. Improved Crossover Operator. The crossover operator affects the global searching performance of the BEGA. Many researchers proposed new crossover mechanisms to promote the global searching performance of the BEGA. Zhang Qiongbing et al. proposed a new crossover mechanism called Same Adjacency crossover; it solved the optimization problem of variable length path through finding more identical genes and exchange gene fragments between Adjacency points and effectively [31]. Pawel Blazej et al. adapted a crossover operator based position for the most restricted model and developed a new type of crossover operator for the more general models. The method could significantly improve the quality of the solutions in the problem of optimization of the genetic code [32]. Yao-Chen Chuang et al. developed a parallel-structured real-coded genetic algorithm (RCGA) with a direction-based crossover operator, named the RGA-RDD. It provided an excellent ability to solve real-parameter optimization problems, especially for those unsolved multimodal and high-dimensional hybrid functions [33].

It is important to select the best crossover operator to pass the excellent gene to the next generation. A common crossover operator is given by (17) in Section 3.2. In this crossover operator, $\alpha$ determines the availability of the excellent genes. It is generally set to 0.5 . However, the fixed value of the parameter $\alpha$ cannot fully utilize the excellent genes. Therefore, an adaptive adjustment strategy is needed to set the value of the parameter $\alpha$. In this paper, the fitness value is selected as the judgment standard of the adaptive adjustment strategy. It is used to reflect the degree of excellence of an individual being tested. Good individuals can be considered as individuals whose fitness values are close to queen. Thus, the adaptive adjustment strategy for the parameter $\alpha$ can be shown as follows:

$$
\alpha=\frac{\text { Fit }\left(\boldsymbol{\theta}_{\boldsymbol{i}}\right)}{\text { Fit (Queen })}
$$

In the equation above, $\alpha$ is determined by the ratio between the current test point and the current optimal value. By increasing the proportion of outstanding individuals, we can retain more genes carried by excellent individuals.

\section{Simulations}

In this section, we demonstrate several numerical simulation results to illustrate the performance of the proposed algorithm. First, comparisons between the conventional BEGA and IBEGA algorithms are shown to verify the effectiveness of the improvements. Second, comparisons among the IBEGA algorithm and other intelligent algorithms are shown to verify the superiority of this paper's algorithm.

In the following simulations, we consider a uniform linear array consisting of $M=16$ identical sensors, and the received signals are narrow-band signals. The sensors are uniformly placed with a spacing of half a wavelength. The number of snapshots is $L=100$, and the interval for the DOA is $\left(r_{\text {min }}, r_{\text {max }}\right)=\left(-90^{\circ},+90^{\circ}\right)$

To evaluate the estimation performance of the different algorithms, we take the Root-Mean-Square Error (RMSE) as our metric $[34,35]$. The RMSE represents the deviation between the estimated value and the true DOA. A lower RMSE represents a higher estimation accuracy. The RMSE is calculated as follows:

$$
R M S E=\sqrt{\frac{1}{P \times P_{\text {run }}} \sum_{j=1}^{P_{\text {run }}} \sum_{i=1}^{P}\left[\widehat{\boldsymbol{\theta}}_{i}(j)-\boldsymbol{\theta}_{i}\right]^{2}}
$$

where $P$ is the number of signal sources, $P_{\text {run }}$ is the number of independent experiments, $\widehat{\boldsymbol{\theta}}_{i}(j)$ is the estimate of the $i$ th DOA achieved in the $j$ th experiment, and $\boldsymbol{\theta}_{i}$ is the DOA of the $i$ th signal source. 
TABLE 1: Parameters of the conventional BEGA and IBEGA algorithms.

\begin{tabular}{lcr}
\hline Parameters & Value & Remarks \\
\hline$l$ & 10 & population size \\
$G_{\text {max }}$ & 500 & maximum generation \\
$\gamma$ & 0.2 & selection ratio \\
$P_{c}$ & 0.8 & crossover probability \\
$P_{m}$ & 0.01 & mutation probability \\
\hline
\end{tabular}

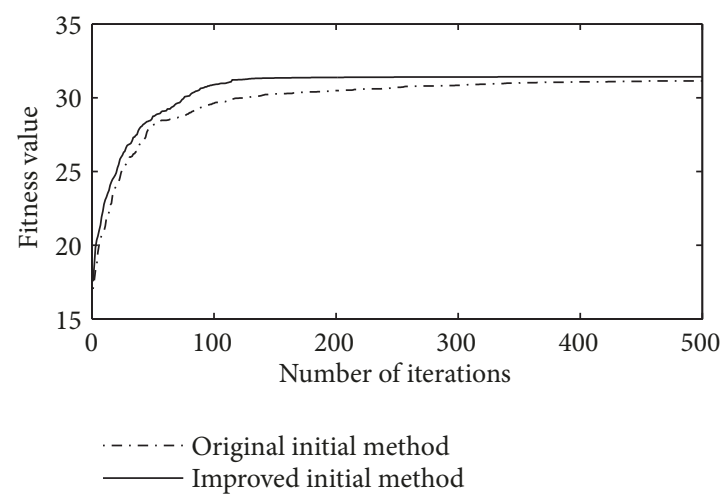

(a) Different population initialization methods

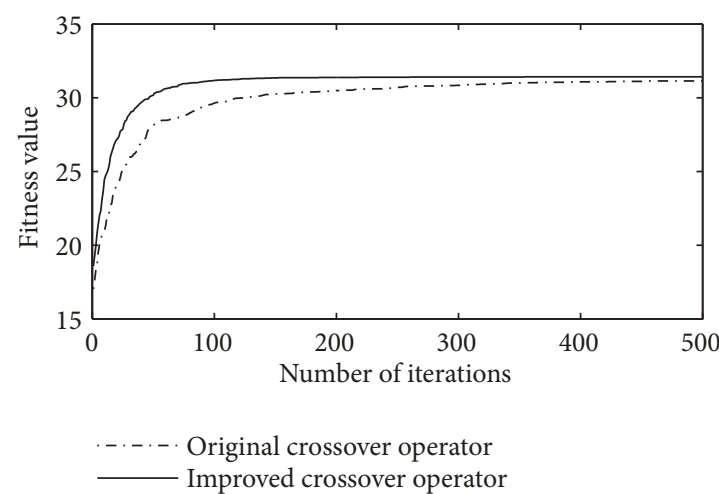

(b) Different crossover operators

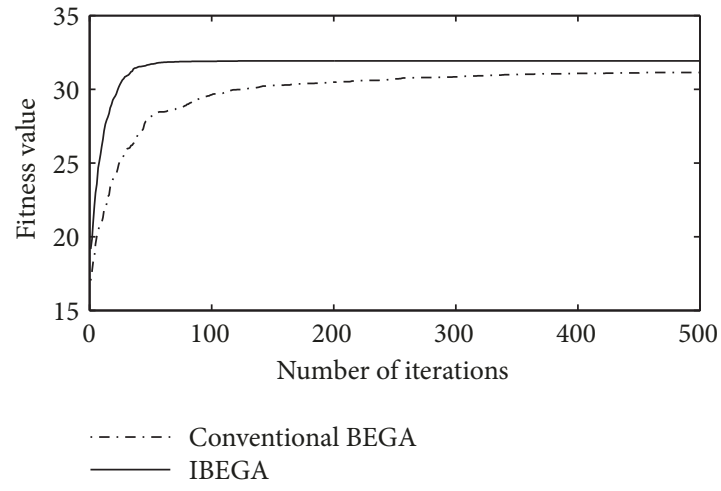

(c) Conventional BEGA versus Improved BEGA

FIGURE 3: Fitness values of the different BEGA-based ML DOA estimations with two signal sources.

4.1. Comparisons between Conventional BEGA and IBEGA Algorithms. In this part, we consider two uncorrelated sources located at $\theta_{1}=-20^{\circ}$ and $\theta_{2}=10^{\circ}$ with a $S N R=$ $0 \mathrm{~dB}$. The parameters of the conventional BEGA and IBEGA algorithms are shown in Table 1.

Figure 3 shows the fitness values of different BEGA-based ML DOA estimations. The fitness values are obtained from an average of 100 experimental runs. Figure 3(a) shows the fitness value comparison between the conventional BEGA and the original and improved initialization methods. Figure 3(b) shows the fitness value comparison between the conventional BEGA with the original and improved crossover operators. Figure 3(c) shows the fitness value comparison between the conventional BEGA and IBEGA algorithms.

As we can observe from Figure 3, each improvement finds an optimum faster than does the conventional BEGA. After comparing Figure 3(a) to Figure 3(b), we can find that the algorithm with the improved crossover operator can find an optimum faster than the algorithm with the improved initialization method. Simultaneously, the fitness value of the optimum found by the algorithm with the improved initialization method or improved crossover operator is slightly higher than that of the conventional BEGA. From the three figures in Figure 3, we can find that the algorithm with both improvements can obtain a faster convergence speed and higher fitness value of the optimum.

From Table 2, we can find not only that the estimation error of each improved algorithm is lower than that of the conventional BEGA but also that this is the case with the RMSE value. Moreover, the algorithm with both improvements obtains the lowest estimation error and RMSE values.

From the above analysis of Figure 3 and Table 2, we find that every improvement of the conventional BEGA proposed in this paper is effective. The IBEGA algorithm proposed in this paper combines the advantages of both improvements; 
TABLE 2: Estimation error and RMSE values under different initialization methods, different crossover operators, and the conventional BEGA and IBEGA algorithms.

\begin{tabular}{|c|c|c|c|c|c|c|}
\hline & & & Estimation error & Average estimation error & RMSE & Average RMSE \\
\hline \multirow{4}{*}{ Initialization method } & \multirow{2}{*}{ Original } & $\theta_{1}$ & 0.42 & \multirow{2}{*}{0.380} & 0.53 & \multirow{2}{*}{0.475} \\
\hline & & $\theta_{2}$ & 0.34 & & 0.42 & \\
\hline & \multirow{2}{*}{ Improved } & $\theta_{1}$ & 0.12 & \multirow{2}{*}{0.120} & 0.13 & \multirow{2}{*}{0.135} \\
\hline & & $\theta_{2}$ & 0.12 & & 0.14 & \\
\hline \multirow{4}{*}{ Crossover operator } & \multirow{2}{*}{ Original } & $\theta_{1}$ & 0.36 & \multirow{2}{*}{0.355} & 0.46 & \multirow{2}{*}{0.440} \\
\hline & & $\theta_{2}$ & 0.35 & & 0.42 & \\
\hline & \multirow{2}{*}{ Improved } & $\theta_{1}$ & 0.07 & \multirow{2}{*}{0.105} & 0.08 & \multirow{2}{*}{0.115} \\
\hline & & $\theta_{2}$ & 0.14 & & 0.15 & \\
\hline \multirow{2}{*}{ Conventional BEGA } & & $\theta_{1}$ & 0.30 & \multirow{2}{*}{0.350} & 0.38 & \multirow{2}{*}{0.445} \\
\hline & & $\theta_{2}$ & 0.40 & & 0.51 & \\
\hline \multirow{2}{*}{ IBEGA } & & $\theta_{1}$ & 0.02 & \multirow{2}{*}{0.070} & 0.03 & \multirow{2}{*}{0.075} \\
\hline & & $\theta_{2}$ & 0.12 & & 0.12 & \\
\hline
\end{tabular}

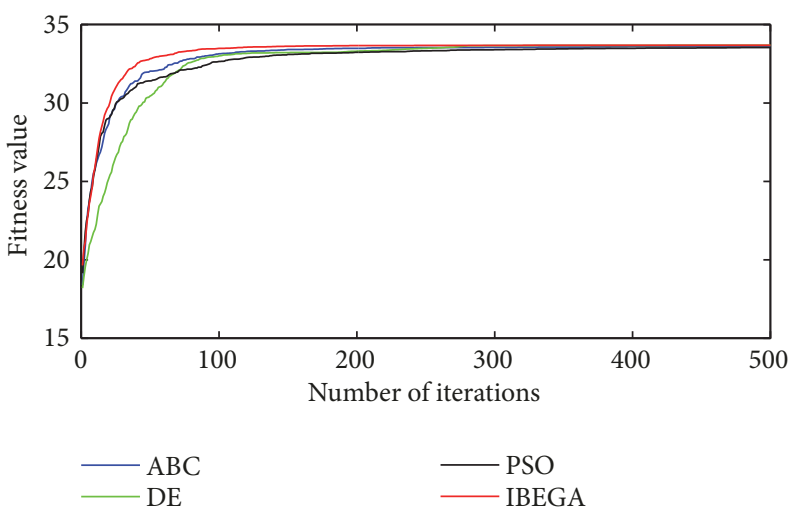

(a) $S N R=0 d B$

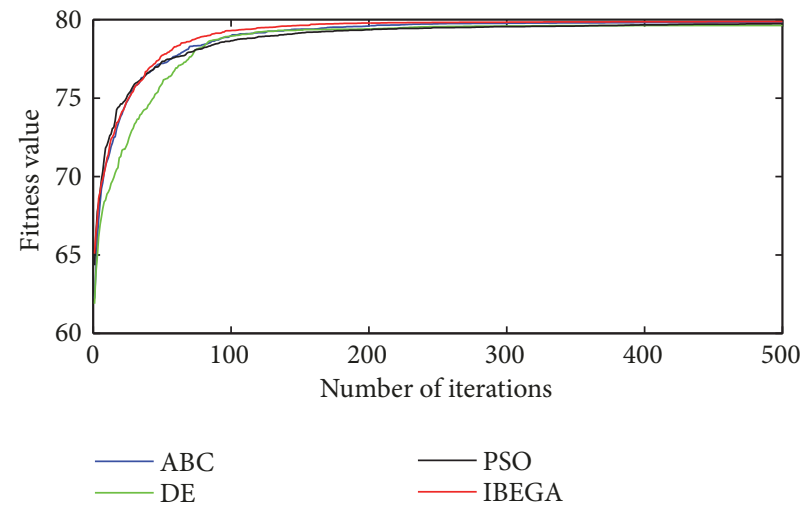

(b) $S N R=-10 d B$

FIgURE 4: Fitness values of ABC, PSO, DE, and IBEGA with two signal sources.

the algorithm can not only obtain a high convergence speed but also retains a high estimation accuracy.

\subsection{Comparisons among IBEGA and Other Intelligent Algo-} rithms. In this part, the convergence property, estimation accuracy, and computational load of the IBEGA-ML, proposed in this paper, are shown by comparison with some intelligent algorithms. These intelligent algorithms are ABC, $\mathrm{DE}$, and PSO. The population size of the algorithms is set to 10 , and the max generation is set to 500 . The other parameters are set properly.

4.2.1. Convergence Property. First, we consider two uncorrelated sources located at $\theta_{1}=-20^{\circ}$ and $\theta_{2}=10^{\circ}$, with $S N R=0 d B$ and $S N R=-10 d B$, respectively. Figure 4 values of $\mathrm{f} A B C, \mathrm{DE}, \mathrm{PSO}$, and IBEGA with two signal sources show the fitness values of ABC, DE, PSO, and IBEGA with two signal sources. The fitness values are obtained from an average of 100 experimental runs. As we can observe, the IBEGA algorithm finds the optimum faster than the other three intelligent algorithms under both cases of $S N R=0 \mathrm{~dB}$ and $S N R=-10 d B$. This means that the IBEGA algorithm can always converge to an optimum within fewer iterations, thereby helping to reduce the computational load.

Then, we consider a scene, whereby the number of signal sources increases. We assume that there are three signal sources in space with $S N R=0 d B$ and $S N R=-10 d B$ at the locations $\theta_{1}=-20^{\circ}, \theta_{2}=5^{\circ}$, and $\theta_{3}=20^{\circ}$. Figure 5 values of f ABC, DE, PSO, and IBEGA with three signal sources show the simulation results. The fitness values are obtained from an average over 100 experimental runs. It is obvious that all four algorithms need more iterations to attain the optimum when more signal sources need to be estimated. However, the IBEGA algorithm still estimates the locations of the signal sources faster than the other three algorithms.

Finally, because the ML DOA estimation method can perform well with coherent signals, we consider two coherent sources located at $\theta_{1}=-20^{\circ}$ and $\theta_{2}=10^{\circ}$, with $S N R=0 \mathrm{~dB}$ and $S N R=-10 \mathrm{~dB}$, respectively. Figure 6 values of $\mathrm{ABC}, \mathrm{DE}$, PSO, and IBEGA with two coherent signal sources show the simulation results. The fitness values are obtained from an average over 100 experimental runs. From the fitness value curves in Figure 6, we find that all four algorithms can still find the optimum of the ML DOA estimation, while the IBEGA algorithm requires the fewest iterations. 


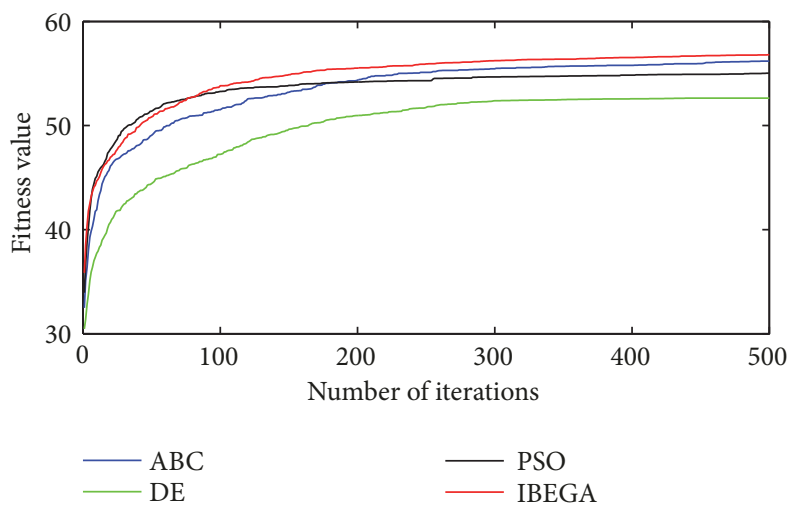

(a) $S N R=0 d B$

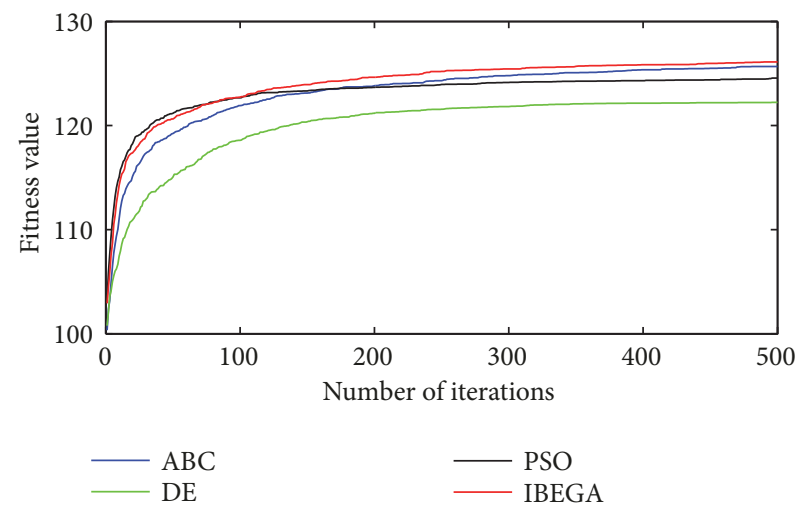

(b) $S N R=-10 d B$

Figure 5: Fitness values of ABC, PSO, DE, and IBEGA with three signal sources.

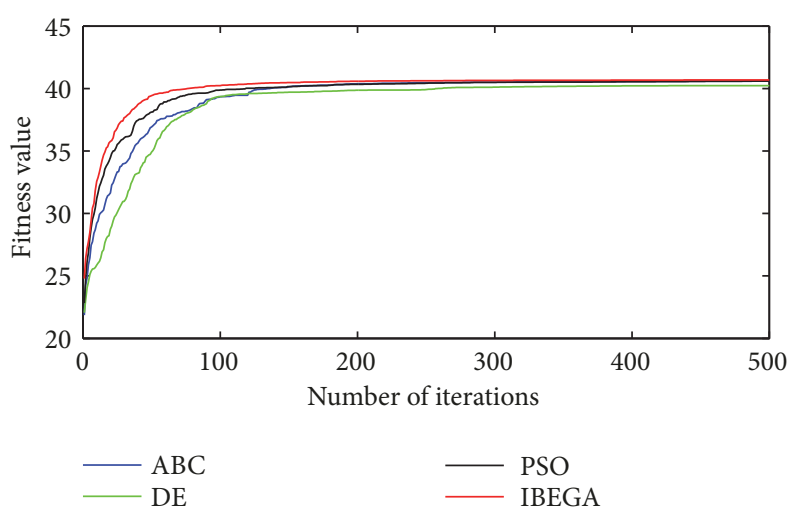

(a) $S N R=0 d B$

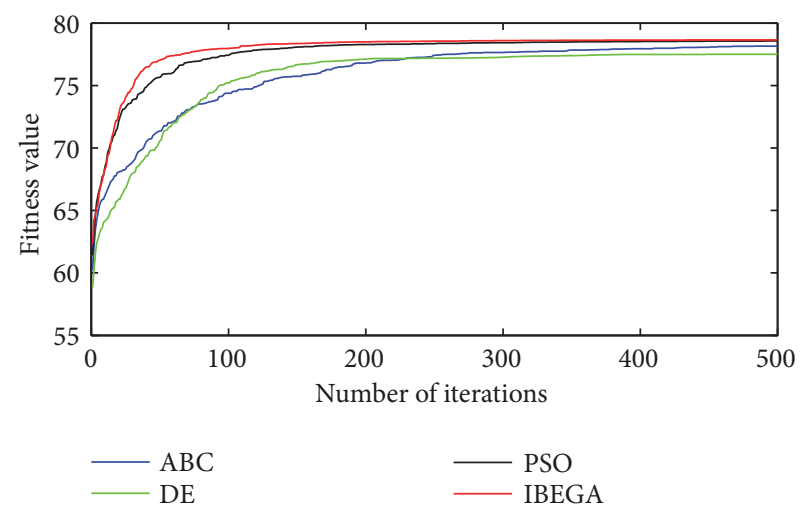

(b) $S N R=-10 d B$

FIGURE 6: Fitness values of ABC, PSO, DE, and IBEGA with two coherent signal sources.

From the above simulation results, we can conclude that the IBEGA algorithm has a higher convergence speed than do the $\mathrm{ABC}, \mathrm{DE}$, and PSO algorithms for the ML DOA estimation problem. The feature determines that the computational load of the IBEGA algorithm is lower than that of the other three intelligent algorithms.

4.2.2. Estimation Accuracy. At the beginning of Section 4, RMSE was also used as the metric to judge the estimation accuracy among the different algorithms, and 100 experimental runs were performed.

Figure 7 shows the simulation results of the RMSE curves for the ML DOA estimations versus $S N R$ with two signal sources and three signal sources. We can observe that the RMSE curve of the IBEGA is slightly lower than that of ABC and is far lower than that of DE and PSO with increased SNR, especially in the scene with more signal sources.

Figure 8 shows the simulation results of the RMSE curves for the ML DOA estimations versus $S N R$ with three coherent signal sources. It is obvious that all four algorithms are effective for ML DOA estimation with coherent signal sources. Meanwhile, the performance of the IBEGA remains better than that of the other three algorithms, especially when the
SNR increases. As the simulation results demonstrated above, we can draw the conclusion that the ML DOA estimation with the IBEGA algorithm performs better than the other three intelligent algorithms, which are used widely.

4.2.3. Computational Load. Equation (14) in Section 2.2 presents the computational load of the ML DOA estimation with the grid search method. With increasing number of signal sources, the computational load increases exponentially when using the grid search method.

The computational load of the ML DOA estimation with the IBEGA algorithm can be expressed as follows:

$$
C_{I B E G A}=\frac{3}{2} \times l \times G_{\text {max }} \times \Delta
$$

where $l$ is the size of the population, $G_{\max }$ is the maximum number of iterations, and $\Delta$ is the number of single DOA calculations.

From (22), we can find that the computational load of the ML DOA estimation with the IBEGA algorithm is independent of the number of signal sources that need to be estimated. It is only dependent on the population size and the maximum number of iterations. From Section 4.2.1, we can find that the IBEGA algorithm needs fewer iterations than 


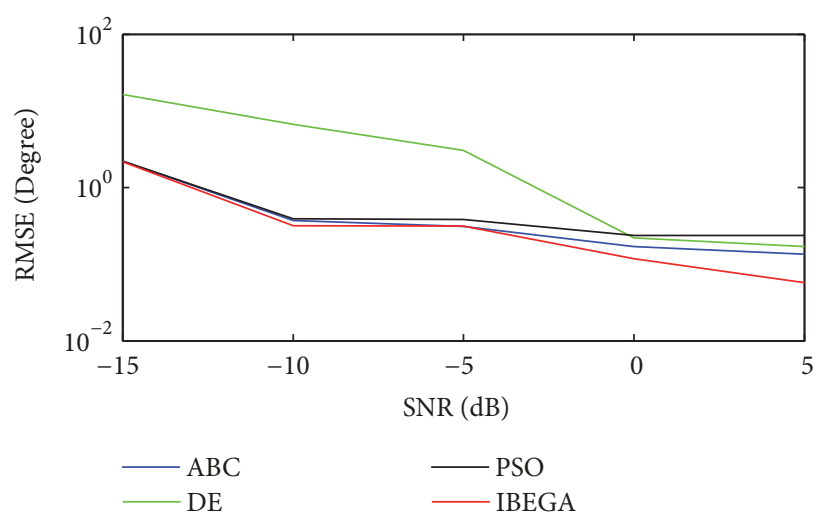

(a) Two signal sources

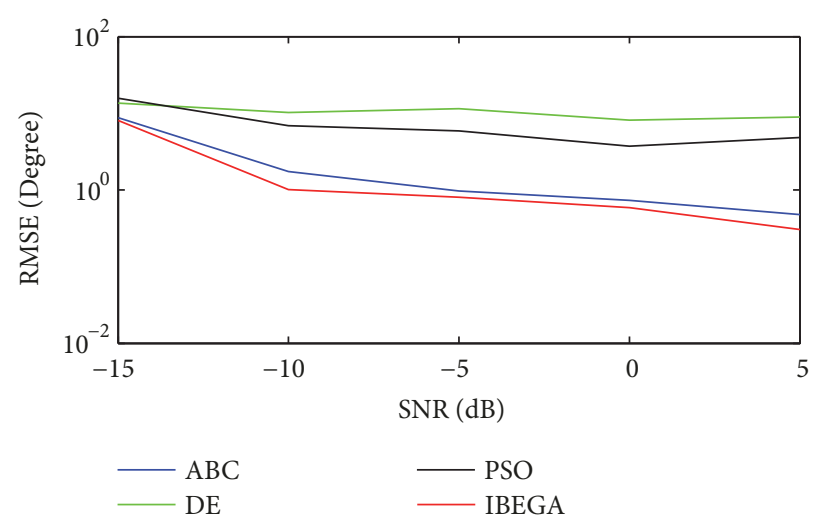

(b) Three signal sources

FIgURE 7: RMSE curves for ML DOA estimations versus SNR for the ABC, DE, PSO, and IBEGA methods with two and three signal sources.

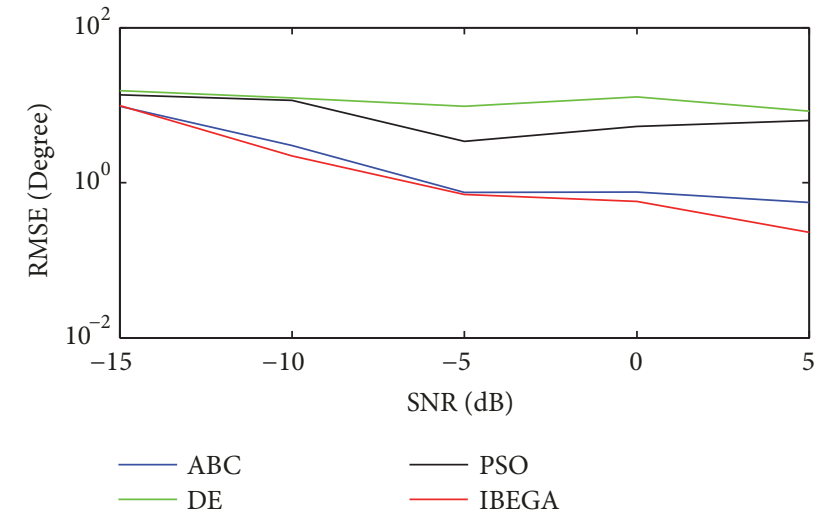

FIGURE 8: RMSE curves for ML DOA estimations versus $S N R$ for the ABC, DE, PSO, and IBEGA methods with three coherent signal sources.

the other intelligent algorithms in all situations. Therefore, we analyse the effects of the population size.

For any intelligent algorithm, the population size affects the computational load because it determines the number of candidate solutions, which is obtained by calculating the maximum likelihood function in each iteration. Thus, an algorithm with a high estimation accuracy and low population size is wanted.

To understand the effects of population size on the estimation accuracy, a situation with two and three signal sources separately with $S N R=0 d B$ is considered. Figure 9 shows the curves of the RMSEs versus the population size of the ABC, DE, PSO, and IBEGA methods. The RMSE values are obtained from an average over 100 experimental runs. From Figure 9, we can observe that the RMSE value decreases with increasing population size. However, when the population size is greater than 10 , the decrease in the RMSE is nonsignificant. When the population size is 10 , the RMSE value is acceptable. Meanwhile, the RMSE value of the IBEGA is the lowest among all four algorithms regardless of the population size.

Now, we consider a situation with two coherent signal sources with $S N R=0 d B$. Figure 10 shows the curves of the RMSEs versus the population size of the ABC, DE, PSO, and IBEGA methods. The RMSE values are obtained over an average of 100 experimental runs. Clearly, the RMSE value under the IBEGA is still the lowest.

Finally, we compare the computation time of the ABC, DE, PSO, and IBEGA methods. Table 3 presents the simulation results. The RMSE values and the computation times are obtained over an average of 100 experimental runs. The population sizes of the four algorithms are set to 10 , and the maximum number of iterations is set to 500. The results are obtained on an Intel Core i3-3220 PC using Matlab 2012a. From Table 3, we can find that not only the computing time but also the RMSE value obtained under the IBEGA is the lowest. This means that under the same estimation accuracy requirement, the IBEGA presents the lowest computational load compared to the other three intelligent algorithms.

Based on the previous analysis, we conclude that the IBEGA algorithm achieves a high estimation accuracy with a low population size.

\section{Conclusion}

In this paper, an improved BEGA algorithm, IBEGA, is proposed to reduce the computational complexity of ML DOA estimation. The BEGA is an improvement on the wellknown GA, and this paper applies it for ML DOA estimation. Although the computational load of the BEGA method is low, its estimation accuracy is unsatisfactory. Therefore, this paper modifies the conventional BEGA algorithm from two aspects: the population initialization method and the crossover operator. All the improvements are verified to be effective according to simulation results. Meanwhile, through comparisons of the IBEGA algorithm to the ABC, DE, and PSO algorithms, which are widely used in the area of DOA estimation, the superiority of the IBEGA algorithm is also verified. Briefly, ML DOA estimation with the IBEGA algorithm can achieve both high estimation accuracy and low computational load. This method provides a reference for related engineering realizations in the area of DOA estimation and is worth further study. 


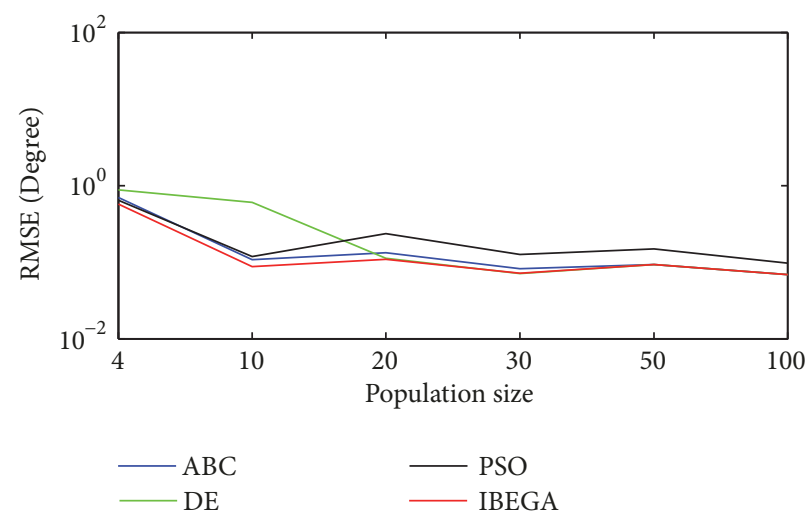

(a) Two signal sources

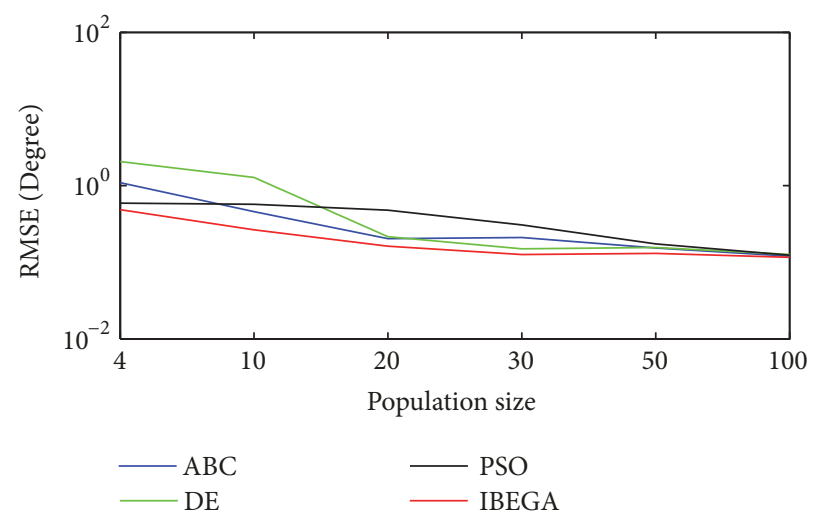

(b) Three signal sources

FIGURE 9: RMSE curves for the ML DOA estimations versus population size for the ABC, DE, PSO, and IBEGA methods with two and three signal sources.

TABLE 3: The computation time of the four algorithms with two and three signal sources when $S N R=0 d B$.

\begin{tabular}{lccr}
\hline Algorithms & No. of Sources & Computation times /s & ${\text { RMSE } /^{\circ}}^{*}$ ABC \\
\multirow{2}{*}{ DE } & 2 & 1.5420 & 0.1675 \\
& 3 & 1.6395 & 0.8781 \\
\hline \multirow{2}{*}{ PSO } & 2 & 1.8417 & 0.1913 \\
& 3 & 1.9043 & 1.1127 \\
\hline \multirow{2}{*}{ IBEGA } & 2 & 1.4759 & 0.3391 \\
& 3 & 1.5717 & 1.3654 \\
\hline
\end{tabular}

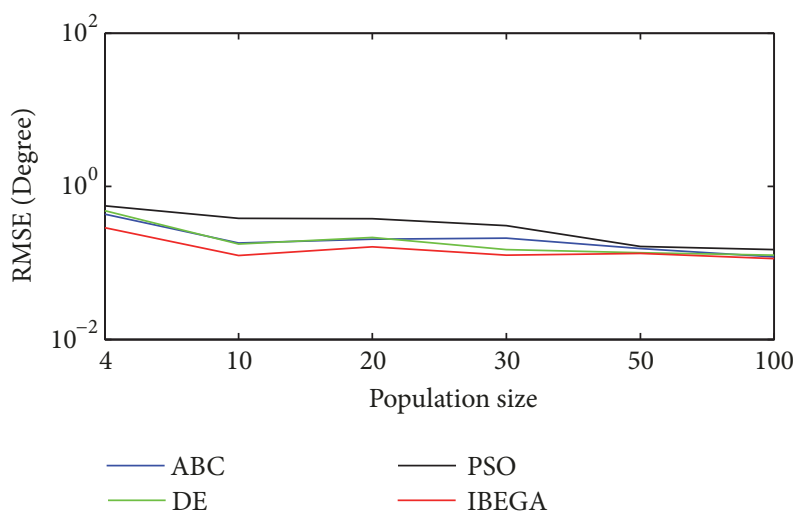

Figure 10: RMSE curves for the ML DOA estimations versus population size for the ABC, DE, PSO, and IBEGA methods with two coherent signal sources.

\section{Data Availability}

The data used to support the findings of this study are available from the authors upon request.

\section{Conflicts of Interest}

The authors declare that there are no conflicts of interest regarding the publication of this article.

\section{Acknowledgments}

This work was supported by the National Natural Science Foundation of China (no. 61573128, no. 61801169, and no. 61671202), the National Key Research Program of China (no. 2016YFC0401606), and the Jiangsu Province Natural Science Foundation (no. BK20170305), which provide financial aid and support for this paper.

\section{References}

[1] S. Sirianunpiboon, S. D. Howard, and S. D. Elton, "Timedecentralized DOA estimation for electronic surveillance," in Proceedings of the 51st Asilomar Conference on Signals, Systems and Computers, ACSSC 2017, pp. 1137-1141, USA, November 2017.

[2] H. Krim and M. Viberg, "Two decades of array signal processing research," IEEE Signal Processing Magazine, vol. 13, no. 4, pp. 6794, 1996.

[3] S. Zhao, Y. S. Shmaliy, and C. K. Ahn, "Iterative maximum likelihood FIR estimation of dynamic systems with improved robustness," IEEE/ASME Transactions on Mechatronics, vol. 23, no. 3, pp. 1467-1476, 2018.

[4] T. C. Yang, "Deconvolved conventional beamforming for a horizontal line array," IEEE Journal of Oceanic Engineering, vol. 43, no. 1, pp. 160-172, 2018.

[5] R. O. Schmidt, "Multiple emitter location and signal parameter estimation," IEEE Transactions on Antennas and Propagation, vol. 34, no. 3, pp. 276-280, 1986. 
[6] R. Roy and T. Kailath, "ESPRIT-estimation of signal parameters via rotational invariance techniques," IEEE Transactions on Signal Processing, vol. 37, no. 7, pp. 984-995, 1989.

[7] R. P. Lemos, H. V. L. E. Silva, E. L. Flores, J. A. Kunzler, and D. F. Burgos, "Spatial filtering based on differential spectrum for improving $\mathrm{ml}$ doa estimation performance," IEEE Signal Processing Letters, vol. 23, no. 12, pp. 1811-1815, 2016.

[8] S. Chen, Y. Li, and H. Chen, "A monocular vision localization algorithm based on maximum likelihood estimation," in Proceedings of the 2017 IEEE International Conference on Real-Time Computing and Robotics, RCAR 2017, pp. 561-566, Japan, July 2017.

[9] S. Cai, Q. Zhou, and H. Zhu, "A sum-of-squares and semidefinite programming approach for maximum likelihood DOA estimation," Sensors, vol. 16, no. 12, 2016.

[10] Y. Wang and K. C. Ho, "An asymptotically efficient estimator in closed-form for 3-D AOA localization using a sensor network," IEEE Transactions on Wireless Communications, vol. 14, no. 12, pp. 6524-6535, 2015.

[11] J.-W. Shin, Y.-J. Lee, and H.-N. Kim, "Reduced-complexity maximum likelihood direction-of-arrival estimation based on spatial aliasing," IEEE Transactions on Signal Processing, vol. 62, no. 24, pp. 6568-6581, 2014.

[12] P. P. Oteiza and N. B. Brignole, "An evolutionary algorithm applied to inventory control for natural gasoline," Industrial \& Engineering Chemistry Research, vol. 55, no. 51, pp. 13062-13073, 2016.

[13] A. H. Beg, M. Z. Islam, and V. Estivill-Castro, "Genetic algorithm with healthy population and multiple streams sharing information for clustering," Knowledge-Based Systems, vol. 114, pp. 61-78, 2016.

[14] L. Lu, J. Yan, and C. W. de Silva, "Feature selection for ECG signal processing using improved genetic algorithm and empirical mode decomposition," Measurement, vol. 94, pp. 372381, 2016.

[15] G. Abu-Lebdeh, H. Chen, and M. Ghanim, "Improving performance of genetic algorithms for transportation systems: Case of parallel genetic algorithms," Journal of Infrastructure Systems, vol. 22 , no. 4, 2016.

[16] P. Shi, X. Fan, J. Ni, and G. Wang, "A detection and classification approach for underwater dam cracks," Structural Health and Monitoring, vol. 15, no. 5, pp. 541-554, 2016.

[17] Z.-B. Shan, Y.-W. Shi, X.-S. Liu, and Z.-C. Zhang, "DOA tracking of moving targets by artificial bee colony algorithm," Guangxue Jingmi Gongcheng/Optics and Precision Engineering, vol. 23, no. 3, pp. 838-845, 2015.

[18] H. Chen, S. Li, J. Liu, F. Liu, and M. Suzuki, "A novel modification of PSO algorithm for SML estimation of DOA," Sensors, vol. 16, no. 12, 2016.

[19] L. Boccato, R. Krummenauer, R. Attux, and A. Lopes, "Application of natural computing algorithms to maximum likelihood estimation of direction of arrival," Signal Processing, vol. 92, no. 5, pp. 1338-1352, 2012.

[20] Y. Jiao, J. Huang, and J. Han, "Fast Bayesian DOA estimator using continuous ant colony optimization," Beijing Hangkong Hangtian Daxue Xuebao/Journal of Beijing University of Aeronautics and Astronautics, vol. 41, no. 1, pp. 65-70, 2015.

[21] J. Wang, L. Zhu, and S. Zhen, "A genetic algorithm for packing problems based on bee evolutionary selection operator," Journal of Graphics, vol. 35, no. 5, pp. 690-696, 2014.
[22] R. R. Rani and D. Ramyachitra, "Application of genetic algorithm by influencing the crossover parameters for multiple sequence alignment," in Proceedings of the 4th IEEE Uttar Pradesh Section International Conference on Electrical, Computer and Electronics, UPCON 2017, pp. 33-38, India, October 2017.

[23] C. Che, J. Su, and X. Zheng, "Satellite module layout design based on adaptive immune bee evolutionary genetic algorithm," Journal of Convergence Information Technology, vol. 9, no. 6, p. 102, 2014.

[24] Q. Zhang, X. P. Wei, J. J. Su, and C. Che, "Satellite module layout design based on adaptive bee evolutionary genetic algorithm," in Mechanical, Electronic and Engineering Technologies (ICMEET 2014), vol. 538 of Applied Mechanics and Materials, pp. 193-197, Trans Tech Publications, 2014.

[25] W. Meng, X.-D. Han, and B.-R. Hong, "Bee evolutionary genetic algorithm," Tien Tzu Hsueh Pao/Acta Electronica Sinica, vol. 34, no. 7, pp. 1294-1300, 2006.

[26] A. Thammano and W. Teekeng, "A modified genetic algorithm with fuzzy roulette wheel selection for job-shop scheduling problems," International Journal of General Systems, vol. 44, no. 4, pp. 499-518, 2015.

[27] Z. Michalewicz, Genetic Algorithms + Data Structures = Evolution Programs, Artificial Intelligence, Springer, Berlin, Germany, 1992.

[28] D. Bajer, G. Martinović, and J. Brest, "A population initialization method for evolutionary algorithms based on clustering and Cauchy deviates," Expert Systems with Applications, vol. 60, pp. 294-310, 2016.

[29] E. Fatemi-Behbahani, K. Ansari-Asl, and E. Farshidi, "A new approach to analysis and design of chaos-based random number generators using algorithmic converter," Circuits, Systems and Signal Processing, vol. 35, no. 11, pp. 3830-3846, 2016.

[30] W. Wei, J. Zhou, F. Chen, and H. Yuan, "Constrained differential evolution using generalized opposition-based learning," Soft Computing, vol. 20, no. 11, pp. 4413-4437, 2016.

[31] Z. Qiongbing and D. Lixin, "A new crossover mechanism for genetic algorithms with variable-length chromosomes for path optimization problems," Expert Systems with Applications, vol. 60, pp. 183-189, 2016.

[32] P. Błażej, M. Wnętrzak, and P. Mackiewicz, "The role of crossover operator in evolutionary-based approach to the problem of genetic code optimization," BioSystems, vol. 150, pp. 6172, 2016.

[33] Y.-C. Chuang, C.-T. Chen, and C. Hwang, "A real-coded genetic algorithm with a direction-based crossover operator," Information Sciences, vol. 305, pp. 320-348, 2015.

[34] L. Yang, Y. Yang, Y. Wang, and J. Zhuo, "Direction-of-arrival estimation of a modified sparse asymptotic minimum variance approach," Shengxue Xuebao/Acta Acustica, vol. 41, no. 4, pp. 465-476, 2016.

[35] Q. Shen, W. Liu, W. Cui, and S. Wu, "Underdetermined DOA estimation under the compressive sensing framework: A review," IEEE Access, vol. 4, pp. 8865-8878, 2016. 


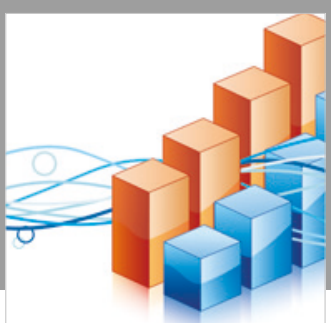

Advances in

Operations Research

\section{-n-m}
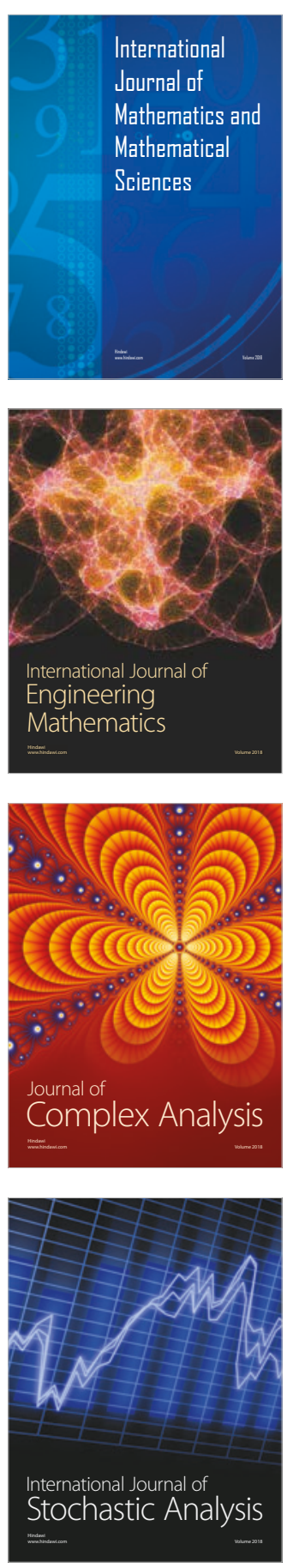
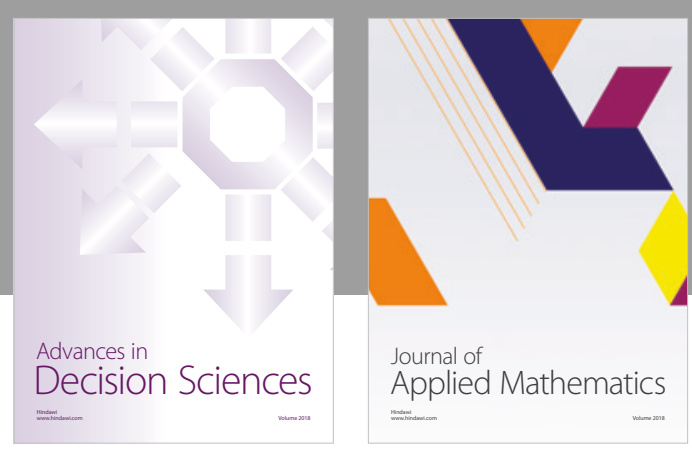

Journal of

Applied Mathematics
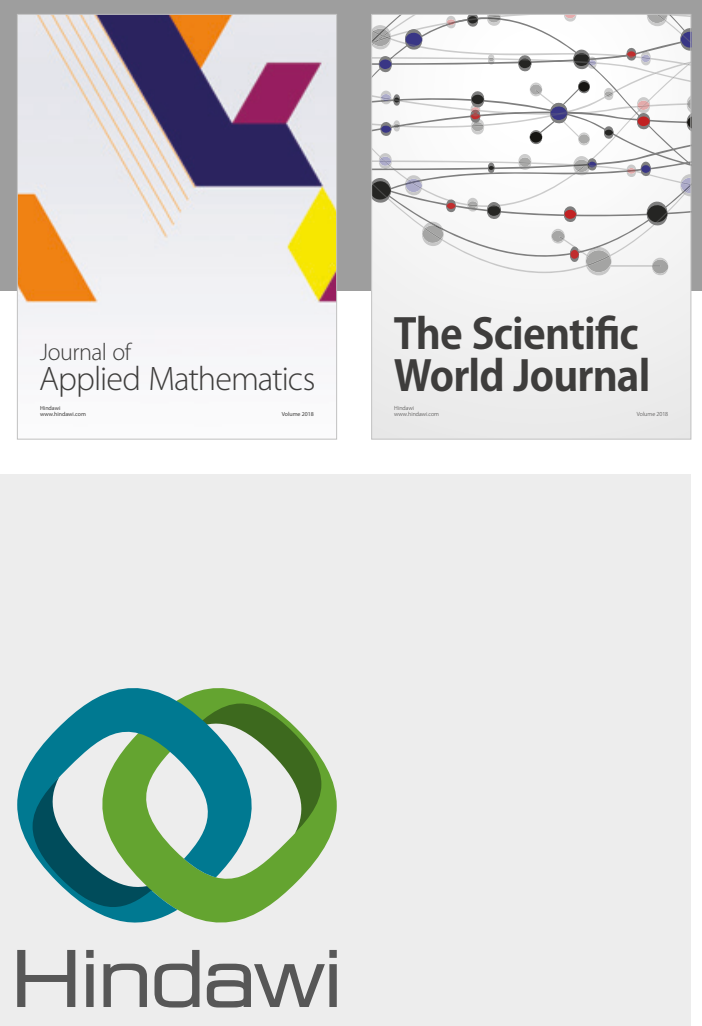

Submit your manuscripts at

www.hindawi.com

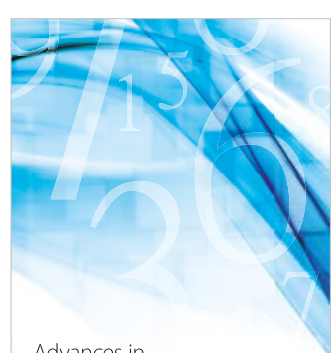

Advances in
Numerical Analysis
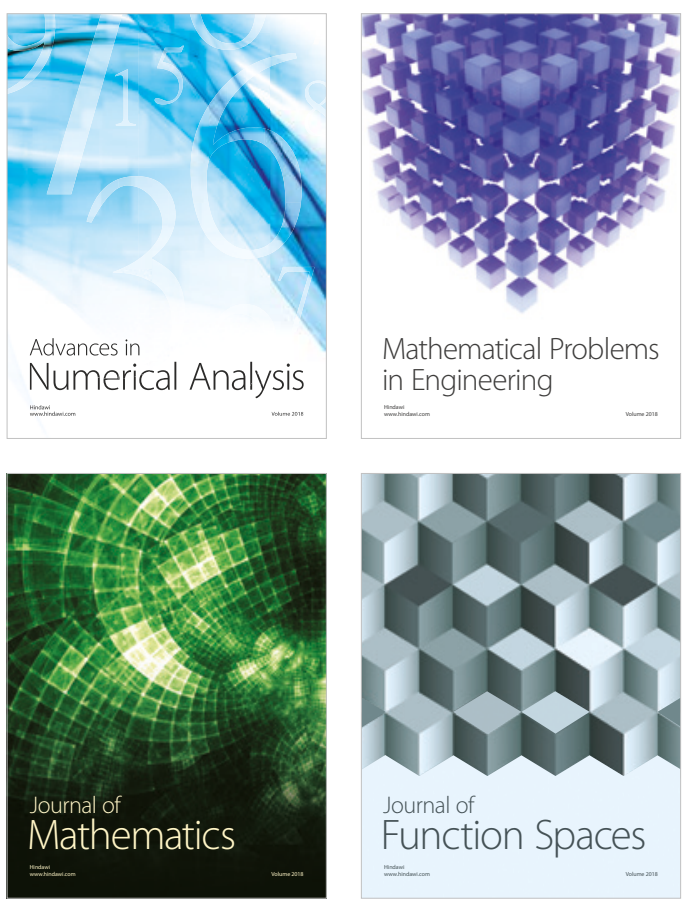

Mathematical Problems in Engineering

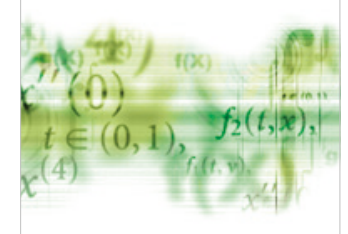

International Journal of

Differential Equations

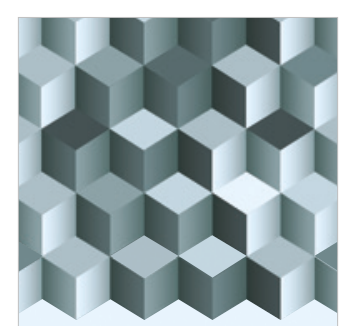

Journal of

Function Spaces

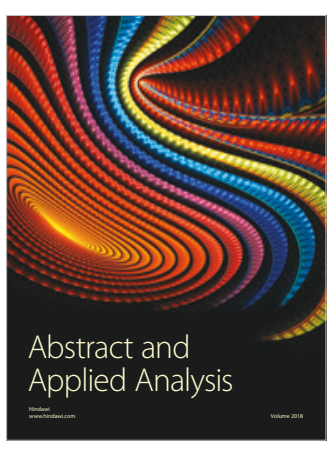

The Scientific

World Journal

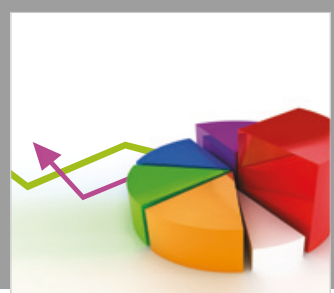

Journal of

Probability and Statistics
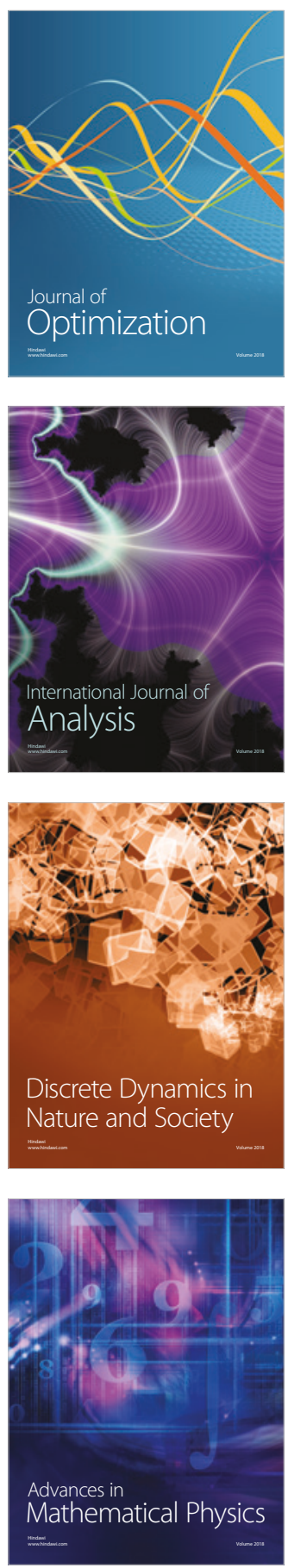